\title{
Effect of hyperthermia on experimental choroidal melanoma
}

\author{
S E PRISCILLA BURGESS,' STANLEY CHANG,' PAUL SVITRA,' \\ JACK DRILLER, ${ }^{2}$ FREDERIC L LIZZI, ${ }^{2}$ AND D JACKSON COLEMAN ${ }^{1}$
}

From 'Cornell University Medical Center, Department of Ophthalmology, 1300 York Avenue, New York, New York 10021, and ${ }^{2}$ Riverside Research Institute, 330 West 42nd Street, New York, New York 10036

SUMMARY Thirty-five rabbit eyes were implanted subchoroidally with Greene's hamster melanoma. When the tumours reached a base diameter of $5 \mathrm{~mm}$, they were treated with ultrasonically induced hyperthermia with a range of temperatures and exposure durations $\left(43-67^{\circ} \mathrm{C}\right.$ and $75 \mathrm{~s}$ to $\left.60 \mathrm{~min}\right)$. Of the 23 treated eyes examined two months after treatment eight showed complete regression of the tumour. Seven showed initial tumour regression, but there was subsequent regrowth of tumour round the margins of the original mass. In eight eyes the tumour continued to grow, though in some cases the rate of growth appeared to be slower than in the controls. In contrast, in all untreated animals the tumour grew to fill the vitreous cavity. These preliminary findings indicate that ultrasonically induced hyperthermia can be an effective local treatment of this intraocular tumour model.

The tumoricidal effect of hyperthermia either alone or in conjunction with radiotherapy is well known.' Heat treatment of tumours has ranged from the use of hot waterbaths ${ }^{2}$ to regional perfusion with warmed blood. ${ }^{3}$ Modern methods of inducing selective heating of tissues has involved radiofrequency electromagnetic waves, ${ }^{4}$ microwaves, ${ }^{56}$ and ultrasound techniques. ${ }^{78}$ This paper describes local, ultrasonically induced hyperthermia (UIH) in the treatment of experimental choroidal melanoma. We describe the model and define temperature and exposure duration relationships required to kill Greene's hamster melanoma growing in the choroid of rabbits.

\section{Material and methods}

Thirty-five eyes of 26 rabbits (New Zealand White and Dutchbelt strains weighing between 2 and $3 \mathrm{~kg}$ ) were studied.

An experimental model of choroidal melanoma was prepared by a modification of Krohn and colleagues' technique. ${ }^{9}$ The tumour stock used was a strain of amelanotic Greene's melanoma ${ }^{10}$ maintained by serial transplantation into the anterior

Correspondence to D Jackson Coleman, MD, Cornell University Medical College. Department of Ophthalmology, 1300 York Avenue, New York, New York 10021, USA. chamber of rabbit eyes. The tumour was dissected from the iris stroma of the freshly enucleated eye and placed in F10 tissue culture medium. It was minced into pieces measuring approximately $1 \mathrm{~mm}^{3}$. Thirty minutes prior to implantation the tumour fragments were removed from the medium and dried on cellulose spears, which reduced the volume and increased the consistency of the implanted tissue. The recipient rabbits were then anaesthetised (intramuscular ketamine $100 \mathrm{mg}$ and xylazine $10 \mathrm{mg}$ ). The eye was rotated inferotemporally with a limbal traction suture. A conjunctival peritomy was made in the superonasal quadrant, and a small scleral flap was dissected approximately $6 \mathrm{~mm}$ posterior to the limbus, forming a pocket. The incision was deepened to expose the choroid. Tumour fragments were inserted into the subchoroidal space with a 20 gauge lumbar puncture needle with a polished tip and recessed trocar. The scleral flap was closed over the tumour with a running suture and the operative field irrigated with water to remove or lyse any free tumour cells on the episclera. Tumour growth in the choroid was monitored by indirect ophthalmoscopy.

Ten rabbits underwent phacoemulsification prior to tumour implantation. This improved visualisation of the tumour through the cornea while the eye was immersed in the waterbath during treatment. Refrac- 


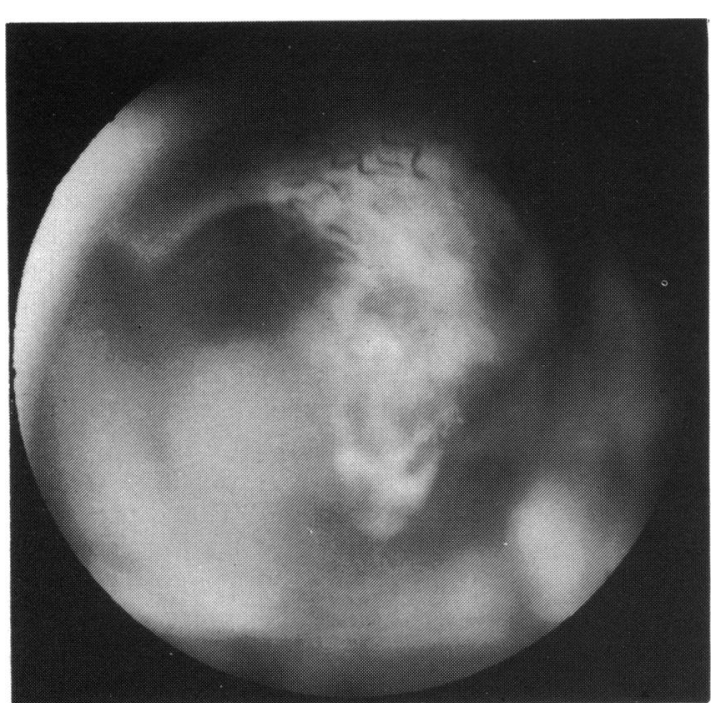

Fig. 1 Fundus photograph showing elevated mass of Greene's melanoma in rabbit choroid prior to treatment.

tive and absorptive effects of the lens on the ultrasound beam were avoided, and the growth of the tumour into the vitreous was unimpeded by the large lens of the rabbit.

The tumours took an average of two weeks to attain a base diameter of approximately $5 \mathrm{~mm}$ and a height of $3 \mathrm{~mm}$. At this stage the tumour was judged to be suitable for treatment. As the tumour grew, it was usually surrounded by a localised exudative retinal detachment or retinal neovascularisation (Fig. 1).

A therapeutic ultrasound system designed specifically for ophthalmic use" was employed. The therapeutic transducers were spherically curved shells. Two transducers were used, one of diameter $42 \mathrm{~mm}$ and the other of $80 \mathrm{~mm}$. Each had a $90 \mathrm{~mm}$ focal length and was operated at its third harmonic (4.6 MHz). Included in this assembly was a coaxial 7.5 $\mathrm{MHz}$ diagnostic ultrasound transducer used to obtain biometric data and a fibreoptic light source for visual orientation of the centre of the ultrasound beam. A coupling bath arrangement was used to provide low loss transmission between the eye and ultrasound transducer by means of a plastic drape slipped over the proptosed eye of the anaesthetised rabbit. ${ }^{12}$ The therapeutic transducer was manually positioned, and the desired distance from transducer to the surface of the tumour was read from the diagnostic A scan.

The temperature was monitored throughout the procedure by means of copper-constantan thermocouples mounted in 29 gauge needles. The thermocouples were connected to a digital thermo- meter module (Bailey Instruments BAT-8). Three thermocouples were used during each treatment. The reference thermocouple was inserted under direct visualisation through the sclera into the tumour centre. A second thermocouple was inserted into the sclera overlying the tumour base in the aphakic rabbits. In the phakic rabbits it was inserted into the sclera opposite the tumour in the region of the exit of the ultrasound beam from the eye. A third thermocouple was placed in the coupling bath. The tumour temperature was kept constant throughout the procedure by monitoring the output of the reference thermocouple and making appropriate adjustments in the excitation voltage applied to the ultrasound therapeutic transducer. Temperature readings from all three thermocouples were taken every minute during treatment. The tumour temperature showed only minor fluctuations during therapy; the average variation from the desired temperature was $\pm 0.9^{\circ} \mathrm{C}$. The average temperature gradient from tumour to sclera in the aphakic rabbits was $4.3^{\circ} \mathrm{C}$ with a range of $1-13^{\circ} \mathrm{C}$. In the phakic rabbits the average temperature gradient across the eye was $6.2^{\circ} \mathrm{C}$, with a range of $3-9^{\circ} \mathrm{C}$.

The coupling bath medium consisted of normal saline initially heated to $37^{\circ} \mathrm{C}$ and allowed to cool during the procedure. The $80 \mathrm{~mm}$ transducer was positioned so that its focal point was situated $12 \mathrm{~mm}$ in front of the anterior tumour surface in the aphakic rabbits (10 eyes). This midvitreous focusing provided a relatively uniform intensity across the width of the tumour; the effective (half power) beam width at the site of the tumour was $10 \mathrm{~mm}$ (Fig. 2A). In the phakic rabbits (17 eyes) the $42 \mathrm{~mm}$ transducer was used with a lateral orientation, and the focal point was positioned approximately $12 \mathrm{~mm}$ external to the sclera (Fig. 2B). This arrangement reduced the dose of ultrasonic energy to the lens.

Treatment regimens used tumour temperatures ranging from 43 to $67^{\circ} \mathrm{C}$ and exposure durations of 75 seconds to 60 minutes. These values were based on the tissue destruction results of in-vivo experiments with local hyperthermia in the treatment of other mammalian tissues. ${ }^{13}$

Eight eyes were implanted with tumour to serve as controls and shams. The four controls were kept for observation following implantation. The other four eyes served as shams - that is, they were given sham hyperthermia treatments. Under anaesthesia the implanted eye was proptosed, surrounded by the saline bath, and the thermocouples were inserted. This configuration was maintained for one hour, but no ultrasound treatment was delivered.

Controls, shams, and treated animals were maintained for observation for as long as three months after tumour inplantation. The effects of treatment 


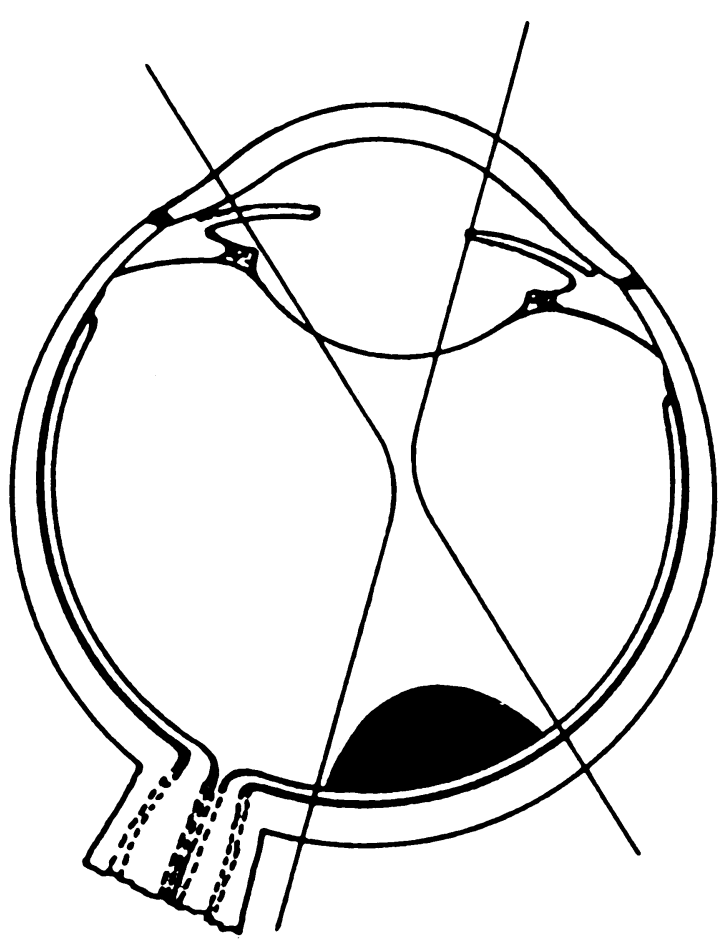

Fig. 2a Arrangement of ultrasound beam in relation to tumour in an aphakic rabbit.

were assessed daily during the first week, twice weekly for a month, and then weekly until the animal was killed.

\section{Results}

In all animals in the control and sham groups, the tumour grew to fill the vitreous space without regression.
In the treated eyes minimal changes were observed immediately following therapy, most often slight blanching of the tumour with occasional subretinal haemorrhage. During the first week after treatment most eyes developed localised subretinal haemorrhage on the surface of the tumour, and the preexisting exudative retinal detachment increased. Small vitreous haemorrhages, which usually did not prevent further fundus observation, were seen in a few animals.

During the second week after treatment it became possible to distinguish three main responses to hyperthermia. In the responsive group (eight eyes) the tumour shrank with resolution of the exudative detachment and haemorrhage. The site of the tumour eventually appeared as a flat area of chorioretinal atrophy. In the initially responsive group (seven eyes) there was an early response with regression of the tumour to a flat scar, but recurrence at the margin of the original tumour mass was seen up to six weeks after treatment. In the non-responsive group (eight eyes), the tumour mass continued to grow. In some of these eyes the rate of growth of tumours appeared slower than the rate of growth of tumours in the control and sham groups. The temperature and exposure duration relationships are plotted in Fig. 3 for a series of 23 eyes: the tumour regression patterns observed are noted by different symbols.

Histopathological examination with light microscopy confirmed the funduscopic observations. In successfully treated cases a flat chorioretinal adhesion developed. The retina was atrophic in the region of the tumour and appeared normal in the adjacent areas (Fig. 4). Implantation of the tumour resulted in sclera thinning at the tumour site. The ultrasound intensities used in this study were much lower than in our previous work, where focused ultrasound was used to produce chorioretinal and
Fig. 2b Arrangement of ultrasound beam in relation to tumour in a phakic rabbit.

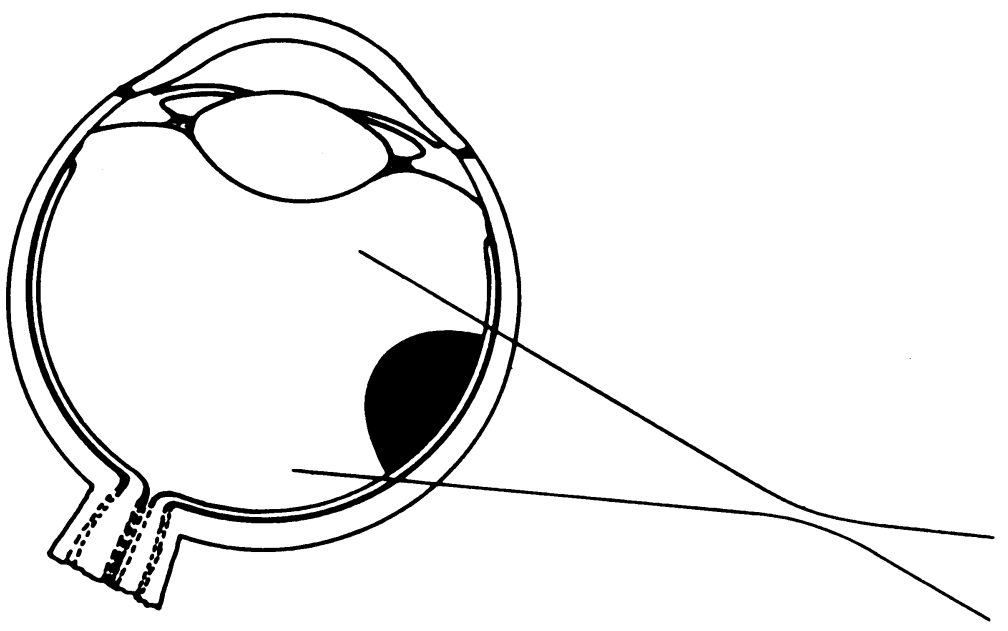




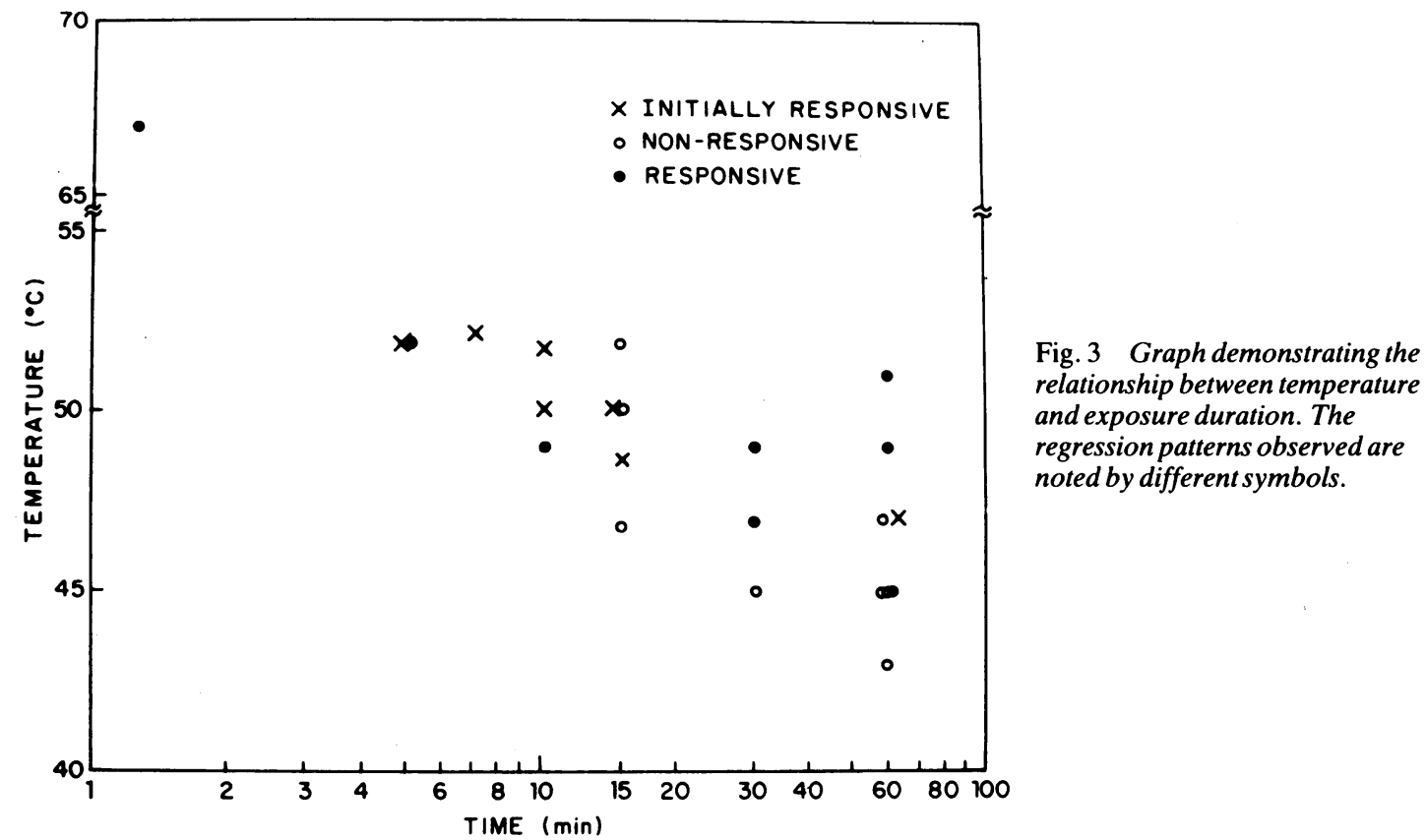

scleral lesions. ${ }^{14}$ Gliosis of the optic nerve was observed in some eyes where the tumour was in close proximity to the nerve head. In non-responsive eyes the tumours contained large areas of malignant cells

mixed with areas of necrosis and haemorrhage. There were numerous mitoses. Scleral infiltration with extrascleral extension was occasionally seen at the site of tumour implantation in sham, control, and

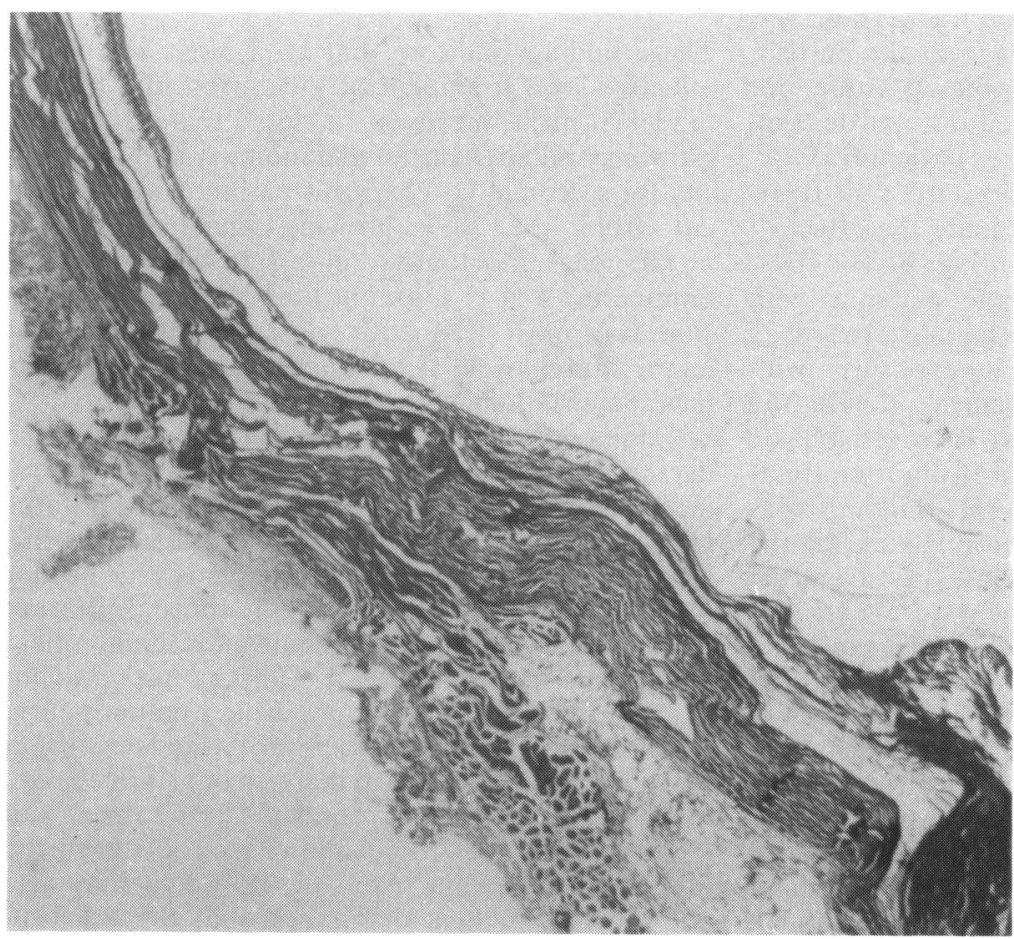

Fig. 4 A flat choroidal retinal adhesion with normal surrounding retina after successful treatment with ultrasound-induced hyperthermia. (Hand E, ×20). 
Fig. 5 Areas of malignant cells with necrosis and haemorrhage in a non-responsive eye. ( $H$ and $E$, $\times 20$ ).

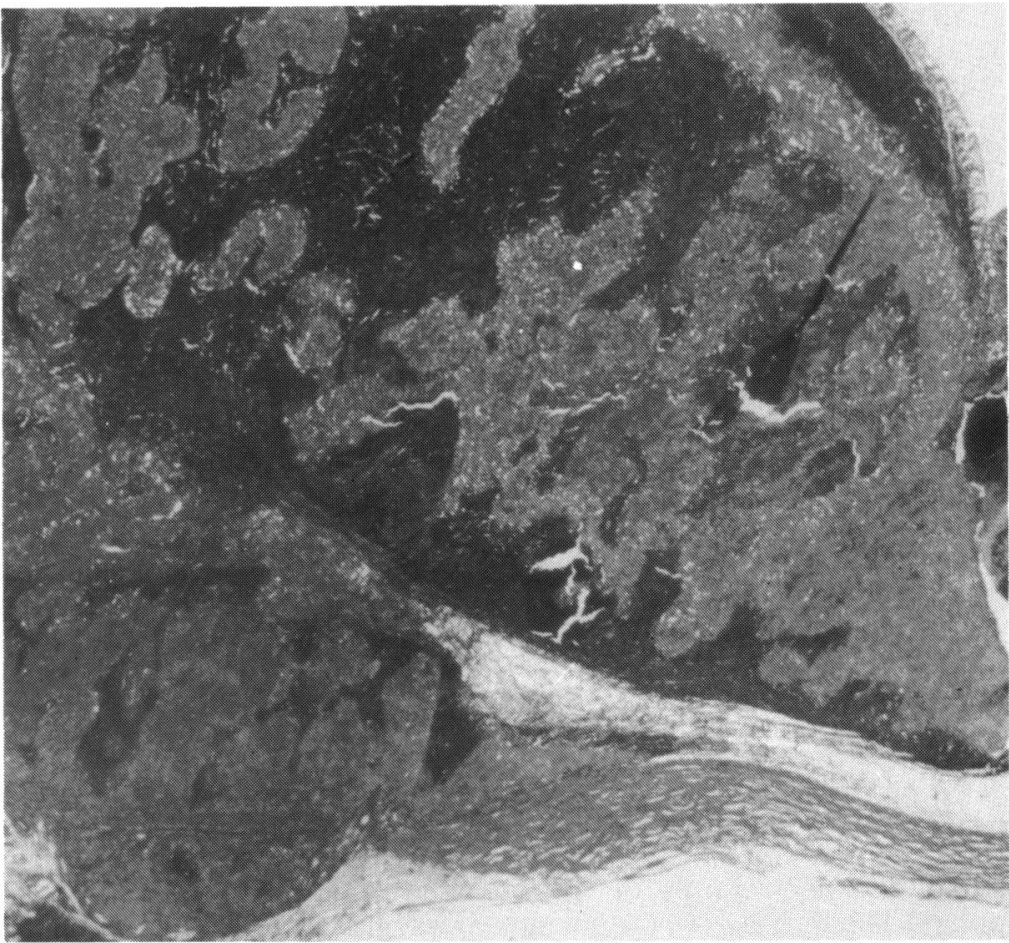

non-responsive eyes (Fig. 5). This was probably due to the presence of the implantation tract. There was no histological evidence of damage to the cornea, iris, or lens in any of the 23 eyes treated with either the 42 or $82 \mathrm{~mm}$ transducer which did not develop immediate post-treatment problems (vide infra).

Four eyes developed complications from the treatment resulting in inability to evaluate the effect of treatment and are not included in Fig. 3. The most serious complication was orbital oedema with secondary proptosis and lagophthalmos, noted 24 hours after treatment. Corneal exposure with resultant ulceration and/or opacification was the outcome in each of these cases. In seven phakic animals with high-temperature short-duration treatment $\left(>49^{\circ} \mathrm{C}, 5-15 \mathrm{~min}\right)$ scleral thinning was observed. In one case scleral melting was seen in the region where the ultrasound beam exited from the eye. Two types of retinal detachment were seen after UIH. In the majority of animals a localised exudative detachment either developed or increased during the first week after treatment. This resolved in the cases where the tumour regressed but increased in eyes containing non-responsive tumours. Seven of the treated phakic eyes developed a rhegmatogenous retinal detachment. As discussed in the next section, these complications were associated with a set of experimental conditions peculiar to the rabbit model.

\section{Discussion}

These findings indicate that UIH alone can be an effective local treatment of this intraocular tumour model. It must, however, be noted that this rapidly growing strain of hamster melanoma with its propensity for sarcomatous change is pathologically different from the slow growing human choroidal melanoma. Initial tumour regression was observed at temperatures of $45^{\circ} \mathrm{C}$ for one hour. Complete regression was most frequently observed at treatment temperatures of $49^{\circ} \mathrm{C}$ for one hour. Other workers have reported that temperatures of $42^{\circ} \mathrm{C}$ to $47^{\circ} \mathrm{C}$ maintained for one hour have resulted in irreversible damage in rodent and rabbit tumours. ${ }^{13}$ Several factors may account for the higher temperatures required to obtain total tumour regression in this work. Most recurrences were observed at the border of the tumour. It is possible that the choroid adjacent to the margin of the tumour could allow greater heat dissipation and establish a temperature gradient between the core of the tumour and its periphery. Thus a subtherapeutic temperature would be present at the tumour borders. Secondly, in our study the eye was proptosed and surrounded by a saline bath initially heated to $37^{\circ} \mathrm{C}$ but allowed to cool during the procedure. The thin sclera of the rabbit may also allow excessive heat dissipation into the adjacent 
waterbath medium. Marginal recurrence of tumour may also be related to inaccurate beam orientation resulting in an edge of the tumour being inadequately heated.

The complications of UIH seen in this study were primarily related to beam orientation. The occasional severe orbital swelling and secondary proptosis were probably a consequence of the anatomical location of the proptosed globe, with subsequent difficulty in the accurate and perpendicular positioning of the ultrasound beam in some animals. Specifically, the proptosed rabbit eye rests against the bony orbital rim, which can result in very high local temperatures for several reasons. Firstly, the orbital bone will reflect virtually all the incident ultrasound, doubling the local intensities and temperature elevations. Secondly, bone, unlike soft tissue, can support ultrasonic shear waves, and the incident compressional ultrasonic wave can be converted to shear waves at the bone surfaces. These shear waves are strongly absorbed by adjacent soft tissues causing very high local temperatures. In the human, acoustic attenuation within the orbital fat will greatly reduce the intensity levels at the orbital wall, and such phenomena should be negligible. Subretinal haemorrhage and exudative retinal detachment, which were observed after treatment in most animals, may have been the result of vascular damage produced by the relatively high temperatures used in this study. The rhegmatogenous retinal detachments seen in seven phakic eyes most likely resulted from inadvertent perforation of the retina by the thermocouple.

It is believed that hyperthermia has multiple actions at a cellular level and on tumour microcirculation. Histologically, tumours treated with hyperthermia show hypertrophy of the Golgi apparatus and an increase in acid-phosphatase-positive lyzosomes six hours after treatment. ${ }^{\text {is }}$ Later this destruction becomes more widespread, as evidenced by derangement of the cytoplasm and rupture of plasma membranes. In addition there is denaturation of enzymes necessary for DNA repair as well as direct inhibition of DNA, RNA, and protein syntheses. ${ }^{16}$

The tumour microvasculature also has an important role in the heat sensitivity of malignant cells. Charateristically the centre of the tumour mass has a sluggish blood flow, whereas in the periphery it may be rapid owing to low tissue pressure and short capillary lengths. Song et al. demonstrated that in normal tissues (skin and muscle of rats) the blood flow increased three- to four-fold when heated to $43^{\circ} \mathrm{C}$ for one hour. ${ }^{17}$ At higher temperatures vascular breakdown occurred. However, tumours heated to these temperatures showed no increase in blood flow but more often a decrease in perfusion. At $45^{\circ} \mathrm{C}$ stasis of blood flow within tumours is observed, with infarction and coagulative necrosis. ${ }^{18}$ Reduction of blood flow may reduce the supply of nutrients to the tumour and slow the egress of cellular breakdown products after hyperthermia. As a secondary effect there is an increase in aerobic glycolysis and lactic acid production. The resulting decrease in intracellular $\mathrm{pH}$ within the tumour may result in activation of lyzosomal enzymes which damage membranes of organelles within the cell.

While this work has evaluated hyperthermia as a sole form of treatment, in current medical practice heat treatment is usually given in combination with radiation therapy. The rationale behind this is the well known synergistic effect between radiation and hyperthermia in the treatment of malignant disease. ${ }^{19}$ This synergism is of potential benefit in ophthalmology, where the most common intraocular tumour, choroidal melanoma, is relatively radioresistant. Focal hyperthermia supplied by ultrasound energy might thus be particularly useful when combined with localised radiation-for example, cobalt plaque to achieve tumour regression with reduced radiation dosage. Initial studies in our laboratory with ophthalmologically relevant human tumour xenographs in athymic nude mice confirms this synergistic effect. ${ }^{20}$

The authors acknowledge the assistance of Dr W Harrison and Dr F Jakobiec with the interpretation of histopathological material and thank Miss Elizabeth Maher for her help in the early stages of this study.

This work was supported in part by NIH Grant EY03174 (DJC) and NIH Grant EY01480 (FLL) and a grant from the Dyson Foundation.

\section{References}

1 Oleson JR, Dewhirst MW. Hyperthermia: an overview of current progress and problems. Curr Probl Cancer 1983; 8: 4-62.

2 Crile G. Selective destruction of cancers after exposure to heat. Ann Surg 1962; 156: 404-7.

3 Cavaliere R, Mondovi B, Moricca G, et al. Regional perfusion hyperthermia. In: Storm FK, ed. Hyperthermia in cancer therapy. Boston: GK Hall, 1983: 369-99.

4 Storm FK, Morton DL, Kaiser LR, et al. Clinical radiofrequency hyperthermia: a review. Natl Cancer Inst Monogr 1982; 61: 343-50.

5 Lagendijk JJW. A microwave heating technique for the hyperthermic treatment of tumours in the eye, especially retinoblastoma. Phys Med Biol 1982; 27: 1313-24.

6 Mendecki J, Friedenthal E, Botstein C. Microwave-induced hyperthermia in cancer treatment: apparatus and preliminary results. Int J Radiat Oncol Biol Phys 1978; 4: 1095-103.

7 Marmor JB, Pounds D, Postic EB, Hahn GM. Treatment of superficial human neoplasms by local hyperthermia induced by ultrasound. Cancer 1979; 43: 188-97.

8 Lele PP. Physical aspects and clinical studics with ultrasonic hyperthermia. In: Storm FK, ed. Hyperthermia in cancer therapy. Boston: GK Hall, 1983: 333-67.

9 Krohn DL, Brandt R, Morris DN. Subchoroidal transplantation of experimental malignant melanoma. Am J Ophthalmol 1970; 70 753-6. 
10 Greene HSN. A spontaneous melanoma in the hamster with a propensity for amelanotic alteration and sarcomatous transformation during transplantation. Cancer Res 1958; 18: 422-5.

11 Coleman DJ, Lizzi FL, Chang S, Driller J. Applications of therapeutic ultrasound in ophthalmology. Progr Med Ultrasound $1981 ; 2: 263-70$.

12 Lizzi FL, Packer AJ, Coleman DJ. Experimental cataract production by high frequency ultrasound. Ann Ophthalmol 1978; 10: 934-42.

13 Dickson JA, Calderwood SK. Temperature range and selective sensitivity of tumors to hyperthermia: a critical review. Ann NY Acad Sci 1980; 335: 180-201.

14 Coleman DJ, Lizzi FL, Jakobiec FA. Therapeutic ultrasound in the production of ocular lesions. Am J Ophthalmol 1978; 86: 185-92.

15 Overgaard J. Histopathologic effects of hyperthermia. In:
Storm FK, ed. Hyperthermia in cancer therapy. Boston: GK Hall, 1983: $163-85$

16 Overgaard J, Skovgaard Poulsen H. Effect of hyperthermia and environmental acidity on the proteolytic activity of murine ascites tumor cells. J Natl Cancer Inst 1977; 58: 1159-61.

17 Song CW, Rhee, JG, Levitt SH. Blood flow in normal tissues and tumors during hyperthermia. J Natl Cancer Inst 1980; 64: 119-24.

18 Eddy HA. Alterations in tumor microvasculature during hyperthermia. Radiology 1980; 137: 515-21.

19 Dewey WC, Freeman ML, Raaphorst GP, et al. Cell biology of hyperthermia and radiation. Meyn RE, Withers HR, eds. Radiation biology in cancer research. New York: Raven Press, 1980: 589-621.

20 Coleman DJ, Lizzi FL, Torpey $\mathrm{JH}$, et al. Hyperthermia in destruction of subcutaneous human tumors in a nude mouse model. J Ultrasound Med 1983; 2 (AIUM Abstract suppl): 37. 